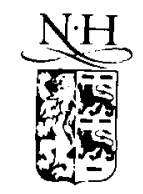

EI SEVIFIR

\title{
Response measurements of a Fabry-Pérot to short pulses
}

\author{
B.J. Offrein, H.J.W.M. Hoekstra, J.P. van Loenen, A. Driessen, Th.J.A. Popma \\ MESA Research Institute, University of Twente, Faculty of Applied Physics, P.O. Box 217, 7500 AE Enschede, The Netherlands
}

Received 15 February 1994; revised manuscript received 25 July 1994

\begin{abstract}
The pulse shape deformation of 80 ps Gaussian input transmitted through a Fabry-Pérot has been determined experimentally and theoretically as a function of the mirror spacing. The effect of mirror surface imperfections is discussed and it is shown that for not too large imperfections there is a strong influence on the transmitted pulse energy whereas the transmitted pulse shape remains almost unaffected.
\end{abstract}

\section{Introduction}

A Fabry-Pérot etalon (FP) is an optical resonator created by two parallel mirrors. Its behaviour originates from the interference of multiple reflections. The way these reflections interfere depends on the roundtrip phase shift $\varphi$; the number of reflections that contribute to the output signal is determined by the losses per roundtrip. The transmission reaches a maximum if the FP is in resonance, i.e. if $\varphi=k 2 \pi$, with $k$ an integer. Around these maxima, the transmission becomes more sensitive for change $\sin \varphi$ by decreasing the roundtrip loss. This sensitivity is used in many applications. A FP for example may be used as a sensor, filter or a modulator. However, the multiple roundtrips make the device principally 'slow'. In case of amplitude modulated beams or short pulses, the response of the etalon may be slow compared to the amplitude variations [1-7]. As presented in literature, the etalon response can be calculated either through a time domain or a frequency domain approach. In these calculations the etalon is treated as ideal, the influence of plate defects is not taken into account. In Refs. [4,5] measurements were reported on the stretching of subnanosecond pulses and re- cently speed of light effects were observed in scanning FP interferometers with a long cavity [8]. In this paper, we compare the measured transmitted pulse energy and shape of 80 ps Gaussian input pulses with theoretical predictions in more detail and discuss the effect of plate defects.

\section{The Fabry-Pérot time response}

In the time domain approach the transmitted field $E_{\mathrm{T}}$ at a time $t$ can be calculated from the incoming field $E_{\mathrm{I}}$ and $E_{\mathrm{T}}$ at $t-t_{\mathrm{r}}$ using:

$E_{\mathrm{T}}(t)=r_{\mathrm{f}} r_{\mathrm{b}} E_{\mathrm{T}}\left(t-t_{\mathrm{r}}\right)+t_{\mathrm{f}} t_{\mathrm{b}} E_{\mathrm{I}}\left(t-t_{\mathrm{r}} / 2\right)$.

Here $r_{\mathrm{f}}, r_{\mathrm{b}}$ and $t_{\mathrm{f}}, t_{\mathrm{b}}$ refer to the front (f) and back (b) mirror field reflection and transmission coefficients, $t_{\mathrm{r}}$ is the photon roundtrip time in the cavity. The frequency domain approach as described in Ref. $[1,3]$ is a good alternative to calculate the pulse transmitted through the etalon. The Fourier transform of the output field is found by multiplication of the FP transfer function with the Fourier transform of the input field; $\bar{E}_{\mathrm{T}}(\omega)=\bar{G}_{\mathrm{FP}}(\omega) \bar{E}_{\mathrm{I}}(\omega)$. The transfer function is given by 
$\bar{G}_{\mathrm{FP}}\left(\omega, t_{\mathrm{r}}\right)=\frac{t_{\mathrm{f}} t_{\mathrm{b}} \exp \left(-\mathrm{i} \omega t_{\mathrm{r}} / 2\right)}{1-r_{\mathrm{f}} r_{\mathrm{b}} \exp \left(-\mathrm{i} \omega t_{\mathrm{r}}\right)}$.

The output pulse in the time domain can be evaluated by inverse Fourier transforming $\bar{E}_{\mathbf{T}}(\omega)$. If the pulse is short compared to the build-up or ring down time (time domain approach) and exhibits a broad frequency spectrum compared to the frequency bandwidth of the etalon transmission peak (frequency domain approach ), then the transmitted pulse will be broadened in the time domain. In Fig. 1, the calculated output pulse is compared to the input for various mirror spacings in the case of a 80 ps Gaussian input pulse incident on a cavity with a finesse of 100. The simulations show a severe broadening of the pulse width accompanied by a long tail.

\section{Experiments}

Experiments are performed using a mode-locked $\mathrm{Nd}$ :YLF laser, generating 80 ps pulses at $1053 \mathrm{~nm}$ with a repetition rate of $76 \mathrm{MHz}$. The etalon was formed by two mirrors with an intensity reflection coefficient of $R=97 \pm 1 \%$. The surface roughness was specified by the manufacturer to be better than $\lambda / N$, with $N=85$. The mirror parallelism could be optimised as the mirrors were mounted in adjustable holders. One holder was mounted on a translation stage for coarse spacing control, fine control was realised with a piezo element. The etalon finesse $(F)$ is defined as the ratio of the free spectral range to the halfwidth of a single transmission peak. The reflectance

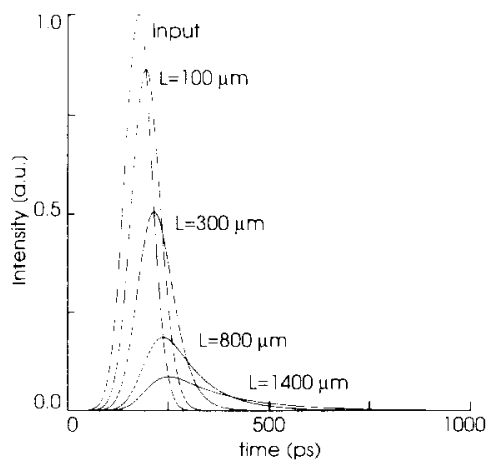

Fig. 1. Calculated deformation of a 80 ps Gaussian input pulse after transmission through a FP with a finesse of 100 for several mirror spacings. finesse $F_{\mathrm{R}}$ is defined by the mirror intensity reflection coefficients:

$F_{\mathrm{R}}=\pi \frac{\sqrt{R}}{1-R}$.

In the case of $97 \%$ reflecting mirrors $F_{\mathrm{R}} \approx 100$. Mirror surface roughness induces roundtrip phase variations across the beam profile from which a defect finesse $F_{\mathrm{D}}$ can be calculated [9]. The reflectance and defect finesse together determine the effective finesse $F_{\mathrm{E}}[9,10]$ as will be measured by scanning the roundtrip phaseshift,

$F_{\mathrm{E}}=\left(F_{\mathrm{R}}^{-2}+F_{\mathrm{D}}^{-2}\right)^{-1 / 2}$.

The effective finesse is directly proportional to the effective half-width of a single transmission peak and was determined to be $F_{\mathrm{E}}=38 \pm 10$ by measuring the transmission as a function of the piezo voltage. These experiments were performed with a small mirror spacing of about $50 \mu \mathrm{m}$ to overcome transient effects. Because the measured effective finesse increases (up to $F_{\mathrm{E}}=75$ ) upon a decrease of the beam diameter, we expect the small $F_{\mathrm{E}}$ in the case of larger beam diameters to be caused by an overall departure from flatness of the mirror surfaces. The large beam diameter of about $2 \mathrm{~mm}$ was used in all further experiments.

Shape and energy of the transmitted pulses were measured for various mirror distances with the experimental setup as given in Fig. 2. The laser beam is split into a reference and a signal branch in which the etalon is placed. An extra distance is introduced in the reference branch in order to separate reference and signal pulses in time before they are coupled into

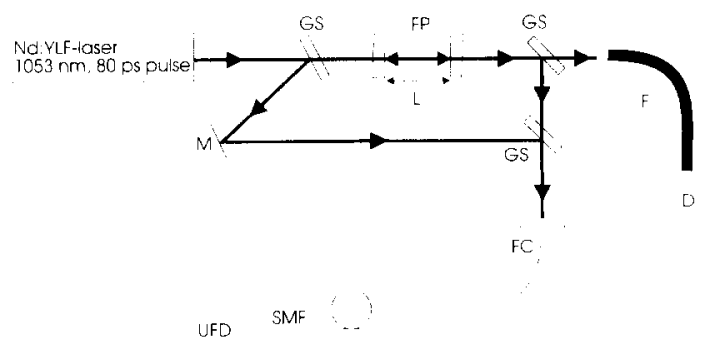

Fig. 2. Experimental setup to determine the pulse shape deformation by the etalon. GS: glass slide, FP: Fabry-Pérot, L: mirror spacing, $M$ : mirror, F: fiber, SMF: single mode fiber, FC: fiber coupler, D: detector, UFD: ultrafast detector. 
a monomode fibre which is connected to an ultrafast diode (New Focus $101 \times$ ). The signal is monitored with a $50 \mathrm{GHz}$ sampling head and sampling scope (Tektronix CSA 803). The average transmitted power was measured with a slow detector. This signal was used to control the resonance condition and determine the transmitted pulse energy decrease with increasing mirror separation. All measurements were performed at maximum energy transmission of the FP and the mirror parallelism was readjusted after every change of the spacing. In Fig. 3a, we present the reference and signal pulses for three spacings. The broadening and deformation of the pulses transmitted by the etalon is clearly to be seen. In Fig. $3 b$ the measured and calculated output pulse shapes are compared. A one parameter fit of the finesse results in a very good agreement with a finesse value of $F=95$. The relative pulse width at half maximum (pwhm) broadening $\left(=\mathrm{pwhm}_{\mathrm{T}} / \mathrm{pwhm}_{\mathrm{I}}\right.$, where the subscripts $T$ and I refer to the transmitted and incoming pulse, respectively) as a function of the nor-

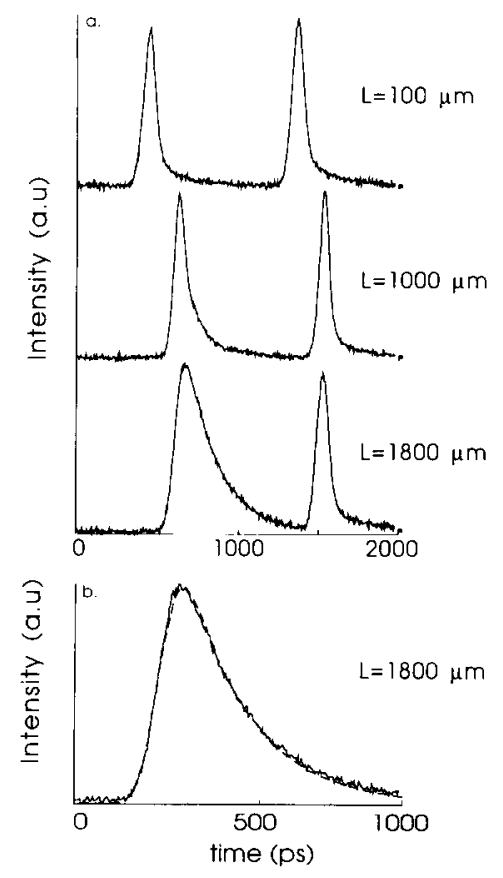

Fig. 3. (a) Experimental pulse shape deformation for various mirror spacings. Left the signal and right the reference pulse. Both signals were adjusted to obtain the same peak voltage. (b) Fit of the calculated and measured pulse shape, one parameter fit of the finesse resulting in $F=95$. malised mirror spacing $\left(t_{\mathrm{r}} / \mathrm{pwhm}_{\mathrm{I}}\right)$ is given in Fig. $4 a$. In the case of small spacings $\left(t_{\mathrm{r}} / \mathrm{pwhm}_{\mathrm{I}} \leqslant 0.03\right)$, it is not clear which finesse value fits with the experimentally obtained broadening. For larger values of the normalised mirror spacing, the broadening fits reasonably with an etalon finesse of about $F=90$, the reflectance finesse. The measured transmission versus mirror separation is depicted in Fig. $4 \mathrm{~b}$ and approaches the $F=90$ curve for large values of the normalised mirror spacing. In Fig. $4 c$, the transmitted pulse energy is normalised with respect to the transmitted energy in resonance at $L=50 \mu \mathrm{m}$. This normalised transmission versus mirror spacing fits to a finesse of about $F=40$, which corresponds to the effective finesse.
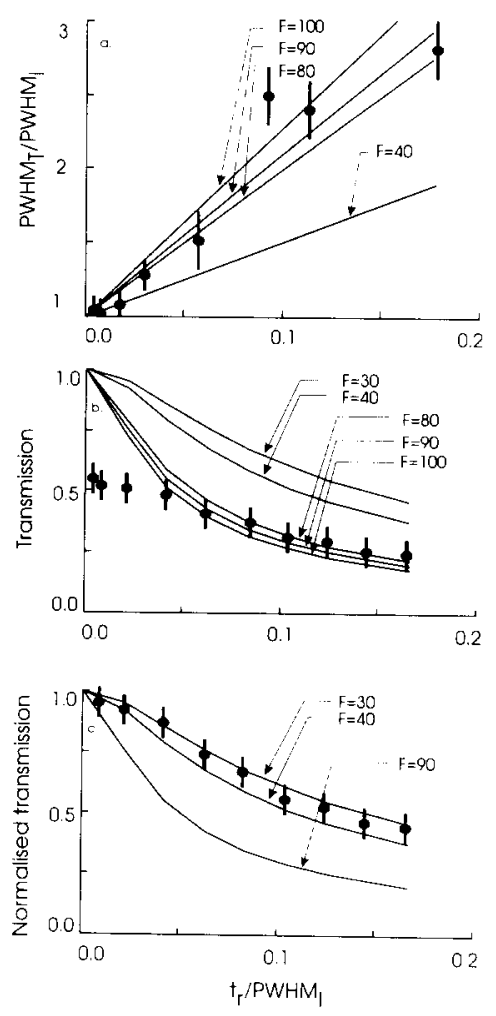

Fig. 4. (a) Measured relative broadening of Gaussian ps pulses as a function of normalised mirror spacing. The solid lines indicate the calculated broadening for various finesse values. (b) Transmission versus normalised mirror spacing, solid lines indicate the transmission as calculated for an ideal etalon. (c) Measured transmission decrease, normalised at the maximum transmittance at $t_{\mathrm{r}} / \mathrm{pwhm}_{\mathrm{L}}=4 \times 10^{-3}$. 


\section{The effect of mirror surface imperfections}

In order to understand the role of $F_{\mathrm{R}}$ and $F_{\mathrm{E}}$ in the pulse deformation and transmission as a function of the normalised mirror spacing, we will take the influence of defects into account. As described by Sloggett [9], surface defects can be modelled with a defect distribution function in the roundtrip phase shift. Equivalently, we describe defects with a defect distribution function in the photon roundtrip time, $d\left(\Delta t_{\mathrm{r}}\right)$, where $\Delta t_{\mathrm{r}}$ is the error in the photon roundtrip time. The contribution of the transmission through the etalon parts with roundtrip time error $\Delta t_{\mathrm{r}}$ is given by $\bar{G}_{\mathrm{FP}}\left(\omega, t_{\mathrm{r}}+\Delta t_{\mathrm{r}}\right) d\left(\Delta t_{\mathrm{r}}\right) \mathrm{d} \Delta t_{\mathrm{r}}$. Hence, the total output is the integral:

$\bar{H}_{\mathrm{FP}}\left(\omega, t_{\mathrm{r}}\right)=\int_{-\infty}^{+\infty} \bar{G}_{\mathrm{FP}}\left(\omega, t_{\mathrm{r}}+\Delta t_{\mathrm{r}}\right) d\left(\Delta t_{\mathrm{r}}\right) \mathrm{d} \Delta t_{\mathrm{r}}$.

As an example of an imperfect FP, we consider the case of nonparallelism of flat interferometer plates across a circular aperture. The defect distribution function is given by [9]

$$
\begin{gathered}
d\left(\Delta t_{r}\right)=\frac{2}{\pi \Delta t_{\mathrm{r}, \max }} \sqrt{1-\left(\frac{\Delta t_{\mathrm{r}}}{\Delta t_{\mathrm{r}, \max }}\right)^{2}}, \\
\text { with }-\Delta t_{\mathrm{r}, \max } \leqslant \Delta t_{\mathrm{r}} \leqslant \Delta t_{\mathrm{r}, \max } .
\end{gathered}
$$

To obtain $F_{\mathrm{E}}=40$ for a $F_{\mathrm{R}}=90$ etalon, the $\Delta t_{\mathrm{r}, \max }$ is found to be $0.04 \mathrm{fs}$, which corresponds to a maximum roundtrip phase variation of $0.02 \pi \mathrm{rad}$. The pulse shape deformation is determined by both the intensity and phase transfer of the etalon. So, to gain insight in the effect of defects on the pulse shape deformation, we compare the intensity and phase transfer function of a $F_{\mathrm{R}}=90 . F_{\mathrm{R}}=90 / F_{\mathrm{E}}=40$ and $F_{\mathrm{R}}=40$ etalon in Fig. 5. For comparison, the intensity transfer of the perturbed etalon is normalised to a maximum of 1 . It is clear that although the full width at half maximum of the normalised intensity transmission resonance of the perturbed $F_{\mathrm{R}}=90 / F_{\mathrm{E}}=40$ etalon is equal to that of the unperturbed $F_{\mathrm{R}}=40$ etalon, the phase transfer is not. In other words, the intensity transfer function can be used to calculate the transmitted energy fraction but is insufficient to model the transient FP behaviour.

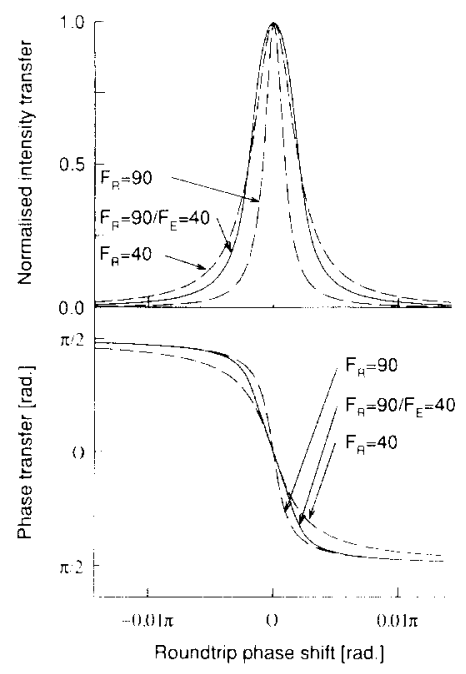

Fig. 5. Intensity and phase transfer functions of an ideal $F_{\mathrm{R}}=90$ etalon, a perturbed $F_{\mathrm{R}}=90 / F_{\mathrm{E}}=40$ etalon and an ideal $F_{\mathrm{R}}=40$ etalon.
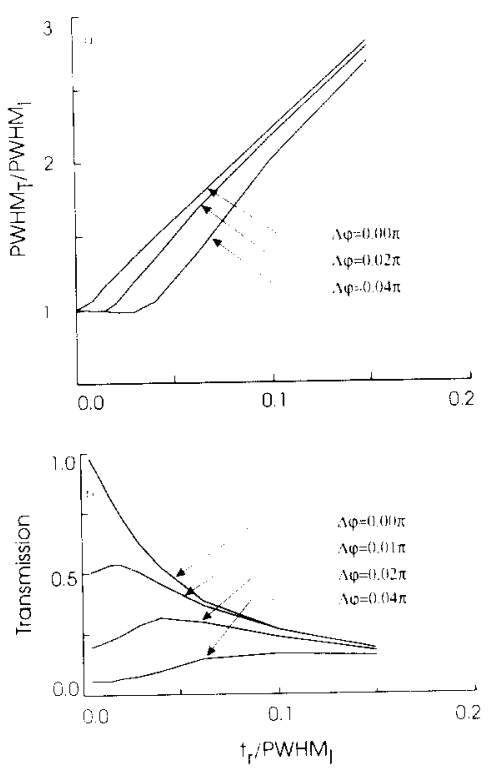

Fig. 6. Calculated relative pulse broadening (a) and transmission (b) versus normalised mirror spacing for various roundtrip phase shifts in the case of a $F_{\mathrm{R}}=100$ etalon.

To show the influence of the defect induced roundtrip time variations (or roundtrip phase shift variations) across the beam profile, we calculate the pulse shape deformation and transmission of an ideal $F_{\mathrm{R}}=100$ etalon for small detuning values, as shown in Fig. 6. Both the transmission and pulse broaden- 
ing show a decrease compared to the in-resonance values. The detuning causes destructure interference of the first transmitted wave with the output after a few round trips, leading to a decrease of transmitted energy and a faster decay of the transmitted pulse. The simulations show that the effect of a small detuning on the pulse width is less pronounced than it is on the transmission. For larger values of $t_{\mathrm{r}} / \mathrm{pwhm}_{\mathrm{I}}$, the influence of detuning errors (mirror defects) decreases and the behaviour approaches the ideal limit. This can be understood as follows. The key quantity to be considered is the number of reflections $(m)$ which constructively contribute to the output. For an ideal FP $m=F_{\mathrm{R}}\left(=F_{\mathrm{E}}\right)$ [2]. For a nonperfect etalon this number is $m_{\mathrm{E}} \approx F_{\mathrm{E}}$, at least for sufficiently large pulsewidths. For pulsewidths for which $\mathrm{pwhm}_{\mathrm{I}} / t_{\mathrm{r}}<$ $F_{\mathrm{E}}\left(t_{\mathrm{r}} / \mathrm{pwhm}_{\mathrm{I}}>0.03\right)$ the number of reflections is smaller than $m_{\mathrm{E}}$, so the effects of plate defect induced phase mismatch will not occur and the behaviour will be that of an ideal FP (see Figs. 4a and b).

The normalised transmission, as presented in Fig. $4 c$, follows from the transmission peak bandwidth alone and is defined by the effective finesse and the mirror spacing. The effective finesse was measured to be $F_{\mathrm{E}}=38 \pm 10$ by scanning the mirror spacing around $50 \mu \mathrm{m}$, which corresponds to the value given in Fig. 4c.

\section{Conclusions}

In conclusion, we measured the FP resonant transmission response to ps Gaussian pulses. Its behaviour can be explained both from frequency and time domain considerations. If the pulses are short compared to the build-up time of the etalon (time domain) and exhibit a frequency spectrum comparable to or broader than the etalon transmission peak (frequency domain) pulse distortion will occur. In this case it is found that the transmitted pulse broadens and obtains a large tail, which increases with increas- ing mirror spacing and is accompanied by a decrease of the transmitted energy. The imperfect etalon response can be described fairly well as an ideal etalon if the number of contributing reflections is smaller than the effective etalon finesse. With a larger number of contributing reflections the transmission deviates from the ideal model prediction but the pulse broadening can still be described by the reflectance finesse, for not too large defects.

The application determines whether the effective or the reflectance finesse limits the etalon performance. If a fast transmission change is important, as in a modulator, the reflectance finesse should be used as the parameter describing the transient behaviour. The effective finesse is the important parameter if the transmitted energy or FP bandwidth is of importance.

\section{Acknowledgements}

The authors thank $H$. van Wolferen, A. Hollink and E. Satink for their valuable contributions. These investigations in the program of the Foundation for Fundamental Research on Matter (FOM) have been supported by the Netherlands Technology Foundation (STW).

\section{References}

[1] C.A. Eldering, A. Dienes and S.T. Kowel, Opt. Eng. 32 (1993) 464.

[2] C. Roychoudhuri, J. Opt. Soc. Am. 65 (1975) 1418

[3] G. Cesini, G. Guattari, G. Lucarini and C. Palma, Opt. Acta 24 (1977) 1217.

[4] W.E. Martin, Optics Comm. 21 (1977) 8

[5] W.E. Martin, Appl. Optics 15 (1976) 3054.

[6] J. Paye, IEEE J. Quantum Electron. 28 (1992) 2262.

[7] J. Paye and D. Hulin, J. Opt. Soc. Am. B 10 (1993) 2372.

[8] K. Hsu, C.M. Miller and J.W. Miller, Optics Lett. 18 (1993) 235.

[9] G.J. Sloggett, Appl. Optics 23 (1984) 2427.

[10] J.M. Vaughan, The Fabry-Perot interferometer (IOP Publishing, Bristol, UK, 1989) pp. 123-127. 